\title{
A Modified and Enhanced Normalized built-up Index using Multispectral and Thermal Bands
}

\author{
Rida Azmi 1*, Omar Bachir Alami², Abd Errahim Saadane ${ }^{3}$, Ilias Kacimi' and Tarik Chafiq ${ }^{4}$ \\ 'Department of Geology, Faculty of Sciences of Rabat, Mohamed V University, Rabat, Morocco; \\ ridaazmi@gmail.com, iliaskacimi@yahoo.fr \\ ${ }^{2}$ Hassania School of Public Works, Casablanca, Morocco; alami.ehtp@gmail.com \\ ${ }^{3}$ Department of Geology, Ecole Supérieure des mines de Rabat, Rabat, Morocco; \\ saadaneabderrahim@gmail.com \\ ${ }^{4}$ Department of Geology, Faculty of Sciences of ben M'sik, Hassan II University, Casablanca, Morocco; \\ Tarik.chafiq1@gmail.com
}

\begin{abstract}
Objective: Mapping impervious surfaces using moderate resolution satellite images is a useful technique for supporting different fields including monitoring and evaluation, planning, statistical analysis and reporting and policy development. Due to the negative impact of impervious surfaces over urban climate, the development of new techniques using spectral indices is a key parameter to extract built up areas with high accuracy. Method: In this study we propose a new concept capitalizing the existing relationships between urban heat island effects and built up areas to produce a modified index, benefiting from the high reflectivity of thermal bands, the mean-infrared and near-infrared band. In addition, spatial enhancement of multispectral data is also used to improve the accuracy of the proposed index, our approach uses spectral reduction of dimensions in order to produce thematic indices (as an input data) instead of continuous images. Finding: Final result showed a high accuracy of built up areas compared to other spectral indices like normalized difference built-up index - NDBI and index -based built-up index - IBI, more than 10\% compared to classic NDBI and $6 \%$ compared to modified index (IBI).
\end{abstract}

Keywords: Landsat OLI, PCA, Remote Sensing, Spatial Enhancement, Spectral Index, Urban Heat Island Effect

\section{Introduction}

Object extraction from spatial remote sensing images is usually performed by two main methods, classification and spectral indices. Each method possesses advantages and inconveniences. In fact, results from traditional approaches of classification to extract built up areas remains laborious and has a high probability of misclassification between bare soil and constructed surfaces; this is due generally to the urban complexity, were spectral reflectance can represent a combination of several land cover called mixed pixels ${ }^{1}$. However, the development of spectral indices helps in the correct interpretation of spatial remote sensing images in various domains, including urban planning, which the success of these indices is due generally to the combination of two or more land surface reflectance with different wavelengths. Several previous studies indicate that spectral indices can be used to extract particularly and effective land features if an appropriate threshold is used ${ }^{2,3}$.

Built-up index is a subset of the spectral indices class, which is one of the most commonly and used approaches for analyzing data in the optical domain. Its interest is seen generally in the fact that urban mapping is based on the extraction of constructed surfaces or impervious areas. It's a very fast process which can be used with any multi spectral sensor property owning a mean-infrared band between 1.55 to $1.77 \mu \mathrm{m}$ and a near-infrared band between 0.76 to $0.9 \mu \mathrm{m}$. This rather important process allows for the spatio-temporal monitoring of the

*Author for correspondence 
urban phenomenon. However, detection of impervious surfaces (which is mainly due to the replacement of bare soil surfaces by constructed ones) is needed to understand the impact of urban phenomenon on the surrounding areas ${ }^{4} \mathrm{Q}$ ihao $</$ author $><$ /authors $><$ /contributor $\mathrm{s}><$ titles $><$ title $>$ Remote sensing of impervious surfaces in the urban areas: Requirements, methods, and trends $<$ / title $><$ secondary-title $>$ Remote Sensing of Environment $<$ | secondary-title $></$ titles $><$ periodical $><$ full title $>$ Remote Sensing of Environment $</$ full-title $><$ / periodical $><$ pages $>34-49<$ /pages $><$ volume $>117<$ / volume $><$ keywords $><$ keyword $>$ Urban remote sensing $</$ keyword $><$ keyword $>$ Impervious surfaces $</$ keyword $><$ keyword $>$ Remotely sensed data characteristics $</$ keyword $><$ keyword $>$ Urban mapping requirements $</$ keyword $><$ keyword $>$ Pixel-based algorithms $</$ keyword $><$ keyword $>$ Sub-pixel based algorithms $</$ keyword $><$ keyword $>$ Object-oriented method $</$ keyword $><$ keyword $>$ Artificial neural networks $</$ keyword $><$ /keywords $><$ dates $><$ year $>2012<$ | ye ar $><$ pub - d ate $s><$ d a te $>2 / 15 /</$ d ate $></$ pub - dates $></$ dates $><$ is bn $>0034-4257</$ isbn $><$ urls $><$ related-urls $><$ url $>$ http://www.sciencedirect.com/science/article/pii/S0034425711002811</ url $></$ related-urls $></$ urls $><$ electronic-resourcenum $>$ http://dx.doi.org/10.1016/j.rse.2011.02.030</ electronic-resource-num $></$ record $></$ Cite $></$ EndNote $>$.

\subsection{Extraction Methods of Built Up Areas}

\subsubsection{Built Up Index Development}

Urban areas are complex ecosystems, in which specialists have tried to divide it into several categories. Therefore, based on some generalized characteristics, (Mirrel K. Ridd 1995) has divided the urban ecosystem into three main constituents, vegetation, impervious surfaces and bare soil ${ }^{5}$. Consequently, he has created (Vegetation Impervious surface and Soil), called VIS model and he considers each pixel on urban areas as a linear combination of three spectra above-mentioned that were chosen to represent the pure surface material in the spectral image.

The detection of impermeability on urban areas requires effective methods to quickly obtain information on built-up surfaces. However, several concepts have been designed to estimate impervious surfaces using remote sensing data. These techniques can be categorized into 4 groups:
$>$ Methods that use visual interpretation or multi spectral classification ${ }^{6}$, considered as manual or semi-automatic classification techniques;

$>$ Classification by integrating the results of impermeability with data from other sources ${ }^{7,8}$;

$>$ Classification based on the spectral mixture analysis method';

$>$ Classification through the relationship between built up areas and other land cover types like vegetation ${ }^{10}$ and bare soil.

The first two methods are mainly applied to the high and very high spatial resolution images, while the last two categories are applied on medium spatial resolution data.

In the footsteps of NDVI, (Zho et al. 2003) developed a multispectral index using moderate resolution image of Landsat 5 Thematic Mapper (TM), it called Normalized Difference Built Up Index - NDBI, the multispectral index is established on the ratio between infrared band with low reflectance on the built surfaces and mean-infrared band with a high reflectance ${ }^{11}$. The author subsequently refined its formula by eliminating disturbances of vegetation through the calculation of NDVI. Classical NDBI used binary images of ( $\llbracket N D B I \rrbracket \Downarrow b$ and $N D V I_{b}$ ) instead of continuous ones, this process reclassified the data assuming that positive values of NDVI and NDBI represent built up, contrariwise, negative values represent the background (Equation 1). Although this reclassification has simplified the interpretation of final results, reference ${ }^{11}$ mentioned that his approach was incapable to distinguish between urban and bare soil areas, this is due to the binarization of the images which limited the refinement of final results.

$$
\text { Built up areas }=N D B I_{b}-N D B I_{b}
$$

NDBI was modified several times using several techniques in order to improve the extraction results. We quote some works, for instance ${ }^{12,13}$, each modified index has advantages and it is applied by following certain distinctive features of the input image. Other researchers combine two or more methods to extract built-up areas ${ }^{14-17}$.

\subsubsection{Advantage of Reducing Dimensions}

The gaps in NDBI approach in terms of refinement final results, is discussed by other studies using spectral reduction of multi-band data. The basic methodology remains the same, but instead of continuous images we can use thematic ones. This technique is informative compared 
to classical counterparts by its special use of derivatives thematic indices to build a new index, more details about it can be found in ${ }^{18,19}$. The derived bands used to build the new index are generally based on the VIS model mentioned in Section 1.1. Based on the creation of a modified built up index, reference ${ }^{19}$ found that ignorance of open waters in the VIS model leads to a noise, which can lead in turn to a number of drawbacks for urban studies. Consequently, researchers were obliged to hide water surfaces before continuing processing in their studies. In addition, the existence of water is very important in urban ecosystems. Otherwise, include open waters as a main component has become essential in the analysis of the bands using spectral indices².

Based on the previous references, we can now generate three thematic indices, including water as a main component. (Normalized Difference Built-up Index - NDBI), (Soil Adjusted Vegetation Index - SAVI) and (Modified Normalized Difference Water Index - MNDWI). The MNDWI is a modification of a water index proposed by $^{20}$ and it's called (Normalized Difference Water Index - NDWI). Accordingly, MNDWI has an advantage over the NDWI by noise suppression of built up areas when applied to open waters. Thus, the three selected indices are expressed in Equations 2, 3 and 4:

$$
\begin{gathered}
N D B I=((M I R-N I R)) /(M I R+N I R)) \\
S A V I=((N I R-R e d) 1+l)) /(N I R+\operatorname{Red}+l))
\end{gathered}
$$

Were $l$ : is a correction factor for soil brightness.

$$
M N D W I=(\text { Green }-M I R)) /(\text { Green }+M I R))
$$

\subsection{Improving Extraction of Built Up Areas}

\subsubsection{Enhancing Special Resolution of Multispectral Data}

Merging two data sets is a fusion technique dealing with the integration of complementary and redundant information from multiple images, the fusion is made in order to create an image composition that contains a better description of the scene $e^{21}$, low and high resolution images can be merged in order to obtain a unique and defined quality of both data ${ }^{22}$. One of suitable techniques used to merge data is the pansharpening method, it's used to improve the quality and the spatial resolution of an image by combining spectral information of low and high spatial resolution imagery. Indeed, the resulting image has a high spectral resolution and the same quality as the high resolution image.
In our case, we assume that spatial enhancement is another important condition to extract and map built-up $\operatorname{areas}^{23}$. However, this condition should preserve useful spectral proprieties of original data, if we use it through a fusion method. Reference ${ }^{24}$ provided a complete review of the most technical methods and references, about 150 academic works on the image fusion, since then, researchers have focused their work on reducing color distortion by improving the quality of fusion techniques. Among the hundreds of existing pansharpening methods, the most popular are: Wavelet based image fusion techniques ${ }^{25}$, Brovey Transform ${ }^{26}$, Principal Component Analysis $^{27}$, Intensity-Hue-Saturation technique ${ }^{28}$.

Despite the performance of the used algorithms, traditional methods are influenced by a significant spectral distortion. Because of the significant difference between the values mentioned of the panchromatic image in the gray scale level and the multispectral images, which is mainly caused by different wavelength ranges. Select an algorithm that does not lead to significant distortion is paramount, because the bias, distortion can falsify the future values, more details about spectral distortion can be found $\mathrm{in}^{29}$.

In a study led by ${ }^{30}$, it was found that a pensharpened image of NDVI values, is highly correlated with those of the original high spectral resolution imagery, indicating that the merger resolutions will not affect spectral properties of the scene, in other words, it causes less spectral distortion.

\subsubsection{Urban Heat Island Effect}

Another important feature of built up areas is the increasing of earth's surface temperature, which is directly related to the urban intensity ${ }^{31}$. This characteristic can be used as an additional indicator characterizing impervious surfaces to map urban areas. In a built-up area, the spectral response of constructed surfaces is slightly different from other land cover types in the heat range of the electromagnetic spectrum, indeed, its use for the characterization of the built up and could add improved extraction results ${ }^{32,33}$.

Our recent works on the city of Casablanca ${ }^{34}$ shows that the heat island effect can be used as an indicator of built up areas, whose, industrial areas shows an elevated temperature compared to surrounding zones. Nevertheless, this characteristic amply that the temperature can be used as an indicator to separate imprevious surfaces from non constructed ones. Other works deal 
with the relationship between land cover types and heat island can be found in ${ }^{35-37}$.

Exploring spatial enhancement of the data and thermal bands in order to enhance buit up index is our primary objective in this study, to develop an enhanced index that can effectively suppress background noise while retaining the basic characteristics of built up using satellite imagery, this is feasible by combining several techniques as reduction of multispectral bands and the benefit from the pansharpening techniques in order to enhance spatial resolution (discussed in Section 1.2), finally operate the thermal sensors to enhance the high reflectivity of built up areas both in the medium infrared and thermal bands.

This paper is organized as follows: Part 1 is a presentation of the study area and the data used for the processing, Part 2 present details for the proposed approch, including analysis of the enhanced index, finaly validation of final result and discussion.

\section{Materials and Methods}

Rabat, Temara and Sale from a conurbation were selected as the study area, of which Rabat-Sale is the administrative center of the region Rabat-Sale-Kenitra. The two cities are located on the Atlantic Ocean at the mouth of the river Bou Regreg whose Sale main as a commuter town. The two cities had a total population of around 577.827 and 890403 inhabitants respectively ${ }^{38}$, about 70\% of inhabitant residing in urban and the rest in peri-urban and rural areas. The study area is a subset of the region Rabat-Sale-Kenitra, covering an area of around $605 \mathrm{~km}^{2}$ and it's characterized by a flat terrain. For the purpose of this research, two cities of this area was used (Figure 1),

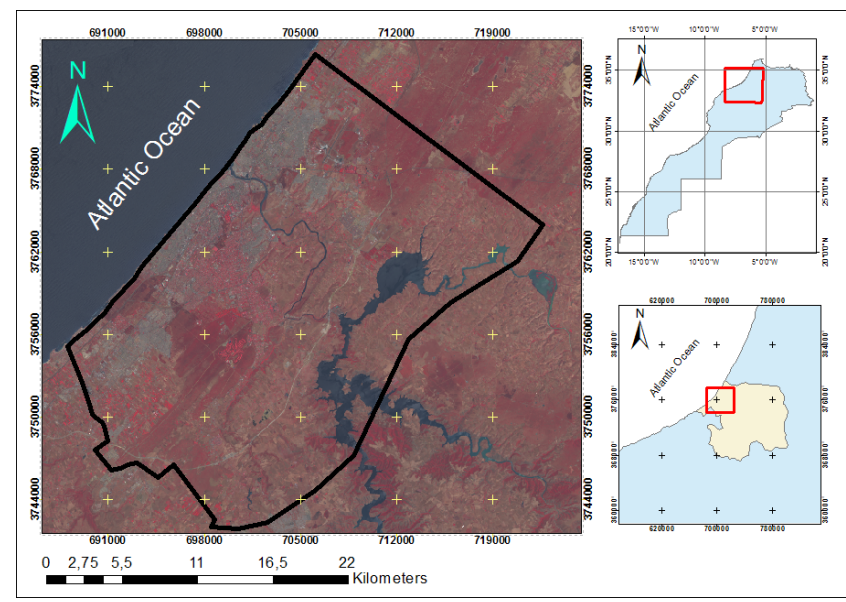

Figure 1. Location of study areas. were selected as they contain areas having built-up densities ranging from very high to very low, bare soil water and other land cover types.

In this paper, we used Landsat 8 OLI and TIRS data, provided by United State of Geological Survey - USGS, Table 1 shows characteristics of OLI and TIRS data, bands (2 to 7) has a spatial resolution of $30 \mathrm{~m}$, whereas that of the thermal bands possess an average resolution of $60 \mathrm{~m}$ resampled to $30 \mathrm{~m}$. Panchromatic band had a spatial resolution of $15 \mathrm{~m}$. Spectral band 1 visible, $(0.43-0.45 \mu \mathrm{m})$ and band 9 cirrus, $(1.36-1.38 \mu \mathrm{m})$ are excluded from further processing.

We proposed a method for automatic extraction of buit up areas composed of three major axes, first, 1. Analyze the dependence between different existing thematic indices generated to calculate the new enhanced index, 2 . Verifies the role of other distinctive features such as the spatial data enhancement, heat islands effect and their contribution in the proposed thematic index, finally 3 . The development of our new approach for semi-automatic extraction of built areas. Figure 2 shows the flowchart of the processing chain used to extract urban areas.

\subsection{Preprocessing}

Preprocessing step involves multi spectral and thermal data, while radiometric correction is paramount, since we will use spectral indices, the variables loyalty with magnitudes of brightness values is required, in other words, the transition from Digital Numbers (DN) to the surface reflectance called Top of Atmosphere (TOA) must be used. For Landsat scenes that are relatively clear, reduced variability between-scene can be attained through standardization for solar irradiance, by converting directy the spectral radiance to reflectance and planetary albedo. This atmospheric reflectance of the Earth are calculated with (Equation 5).

$$
\rho_{p}=\frac{\left(\pi * L_{\lambda} * d^{2}\right)}{E S U N_{\lambda} * \cos \theta_{s}}
$$

Table 1. Characteristics of input data

\begin{tabular}{|c|c|}
\hline Data & Description \\
\hline Sensor type & OLI and TIRS \\
\hline DATE_ACQUIRED & 2015-05-27 \\
\hline Niveau du traitement & Level 1 T $:$ L1T \\
\hline WRS_PATH & 202 \\
\hline WRS_ROW & 36 \\
\hline
\end{tabular}




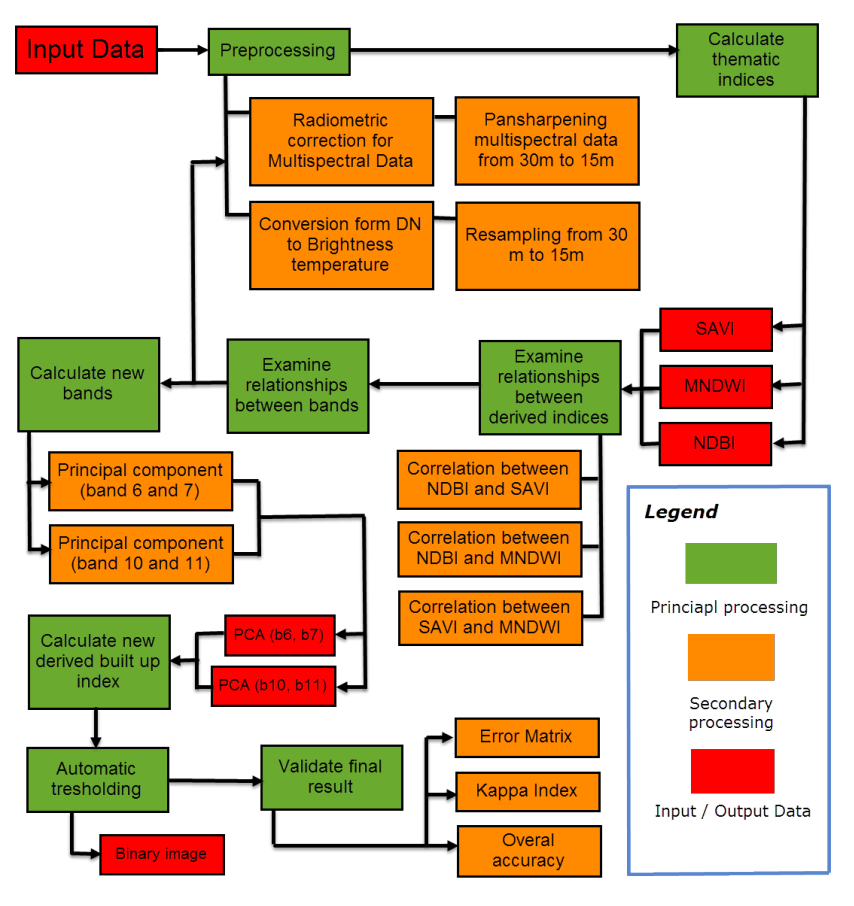

Figure 2. Processing chain.

Where,

$\rho_{p}=$ Top Of Atmosphere - TOA reflectance, which is the ratio of reflected versus total power energy.

$L_{\lambda}=$ Spectral radiance at the sensor's aperture (satellite radiance)

$\mathrm{d}=$ Earth-sun distance in astronomical units. ESU

$N_{\lambda}=$ Mean solar exo - atmospheric irradiance $\theta_{8}=$ Solar zenith angle in degrees, which is equal to $\theta_{8}=90^{\circ}-\theta_{8}$ where $\theta_{8}$ is the sun elevation

As regards using Landsat 8 images, the scenes are provided with specific parameteres at the level of bands, this factor allow direct conversion of DN to the TOA. However, the effects of the atmosphere for instance a disturbance in reflectance that varies with wavelength must be taken into account in order to measure the reflectance at the surface.

We also applied the Dark Object Substraction (DOS) method in order to remove small values. DOS method assumes that if there are very small areas in an image with reflectance values, all the apparent reflectance should be due to the effects of air diffusion and this information can be used to calibrate the rest of the image ${ }^{39}$. it should be noted that the accuracy of DOS method is very useful when no atmospheric measurements are available, but is generally less physically based corrections, the expression developed by ${ }^{40}$ is used in Equation 6.

$$
L_{\downarrow} p=L_{\downarrow} \min -L_{\downarrow}(D O 1 \%)
$$

Were:

$L_{\min }=$ radiance that correspond to a digital count value for which the sum of all the pixels from

$$
\text { the image considered, }
$$

therfore the radiance obtained with that digital count value $\left.\llbracket D N \rrbracket_{\downarrow}(\min )\right)$

$L_{D 01}=$ radiance of Dark object, assumed to have a reflectance value of 0.01

For thermal bands (10 and 11), we converted DN to At-satellite temperature by using the expressed formula of calibration, transformation of thermal data from DN to the brightness temperature in degree celicius without atmospheric correction using the (Equation 7):

$$
\left.T=k_{\downarrow} 2 /\left(\ln K_{\downarrow} 2 / L_{\downarrow} y+1\right)\right)-273.5
$$

Were :

$K_{1}=$ Band - specific thermal conversion constantin in $\frac{\text { whatts }}{\text { metre }}$ squared $*$ ster $* \mu m$ $K_{1} 2=$ Band - specific thermal conversion constant in kelvin) $L_{\lambda}$ in the spectral radiance at the sensor's aperture, mesured in $\frac{\text { watts }}{\text { metres }}$ squared $*$ ster $*$ um

In the case of Landsat data, the formula is expressed in Equation 8:

$$
L_{\downarrow} \lambda=M_{\downarrow} l * Q_{\downarrow} \mathrm{cal}+A_{\downarrow} l
$$

Were:

$M_{l}=$ Band specific multiplicative rescaling factor from Landsat metadata (Radiance Multi ${ }_{\text {Band }}$ ),

where $x$ is the band number)

$A_{l}=$ Band specific additive rescaling factor from Landsat metadata Radiance( $\left({ }_{A D D_{B A N}}\right)$,

\section{where $x$ is the band number)}

$Q_{1}$ cal $=$ Quantized and calibrated standard product pixel value DN)

$\mathrm{K} 1$ and $\mathrm{K} 2$ are constants that we can see the metadata file of input data.

We have not conducted a cloud identification process because the provided image is cloud free image according to the metadata file. 


\subsection{Fusion Methods}

Several studies have shown the importance of enhancing the image spatially to prove its primordial role in the field of classification, automatic and semi-automatic extraction of objects ${ }^{41,42}$. Usually, a multi resolution image fusion algorithm is considered as a good method, when it meets two principal criteria, first is that it must keep the spatial quality of the image, both based on visual inspection of data and performance metrics, secondly, is the preservation of spectral information data. We verified the results from different pansharpening algorithms to find the method that perfectly meets our needs. We evaluated four algorithms considered as best fusion methods:

1. Normalized Color Brovey Pansharpening ${ }^{43}$, 2. Gram Shmidit Pansharpening ${ }^{44}, 3$. Modified IHS method ${ }^{45}$ and 4. Nearest Neighbor diffusion based pansharpening ${ }^{46}$. We carried out a comparison between the original data and the results of different fusion algorithms proposed below, the rule was simple, the method does not produce a large distortion will be the best choice. Therefore, the algorithm NNDifusion and Gram Shmidit algorithm showed low distortion of multi spectral information or the remaining two algorithms have largely distorted the data.

We made a pansharpening of multispectral data based on the panchromatic image of input data using the Nearest-neighbor algorithm pansharpening diffusionbased algorithm. This method proposed by ${ }^{46}$ is generally based on the dissemination of nearest neighbor, to have superior performance in both spatial quality/spectral and computations. The novelty of this approach lies in its functioning by-spectra and the use of a diffusion model to solve the multi spectral fusion problem. The method works locally and is not based on global optimization and can produce identical results regardless of the size of the stage or contained scene. Figure 3 shows the results after the implementation. A limitation of the method in

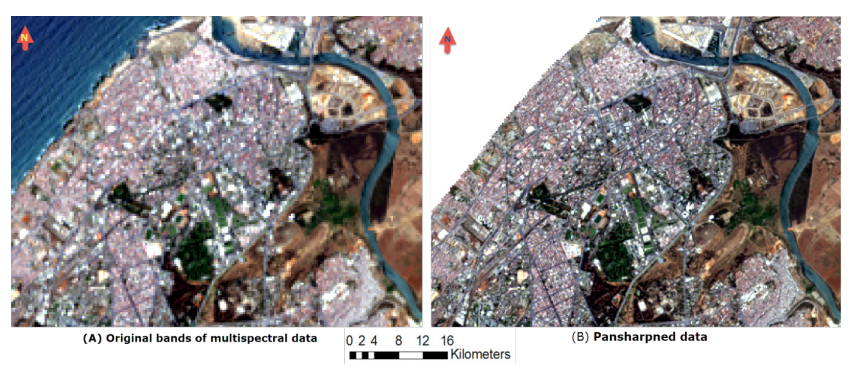

Figure 3. True color composite showing study area before A) and after pansharpening B). the ratio of the merger, when a merger exceeds 1:6, result shows a noise in the visual quality of the data. This will not affect the output data, because the ratio in our case is 1:2. For thermal bands, we used a bicubic method as a resampling technique in order to produce a band with 15 $\mathrm{m}$ instead of $30 \mathrm{~m}$, this technique is suitable for continuous data.

\subsection{Benefit of using Thematic Bands}

We examined the spectral and thermal variations in built-up, vegetation and water using samples of 10 locations in the fused image, multi spectral bands (2-7) and thermal bands (10 -11), DN of each separated classe averaged to draw graphs shown in (Figure 4a). we note

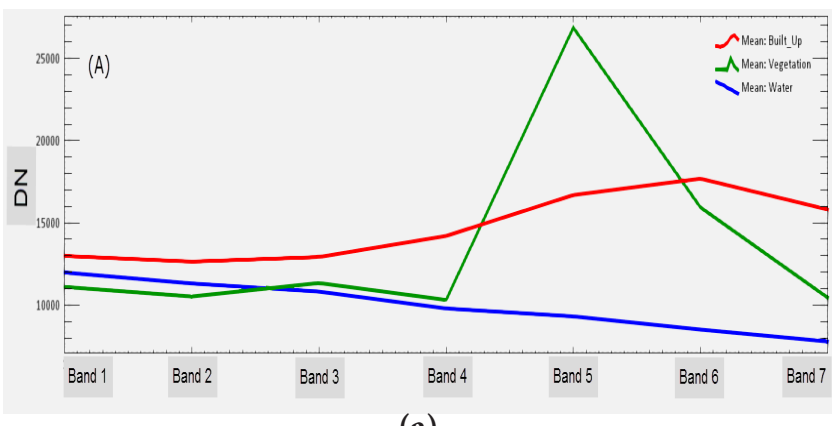

(a)

(B)

Mean temperature

40

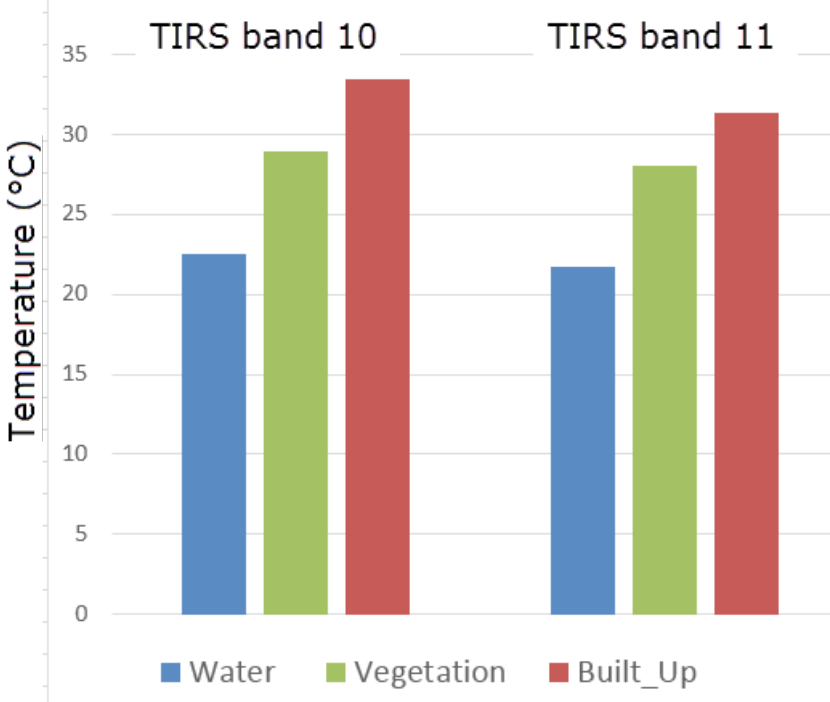

(b)

Figure 4. Reflectance of built-up, vegetation and water areas in A) Optical bands 2-7 DN value) and B) Thermal bands 10-11 temperature in degree Celsius) of Landsat-8 OLI image. 


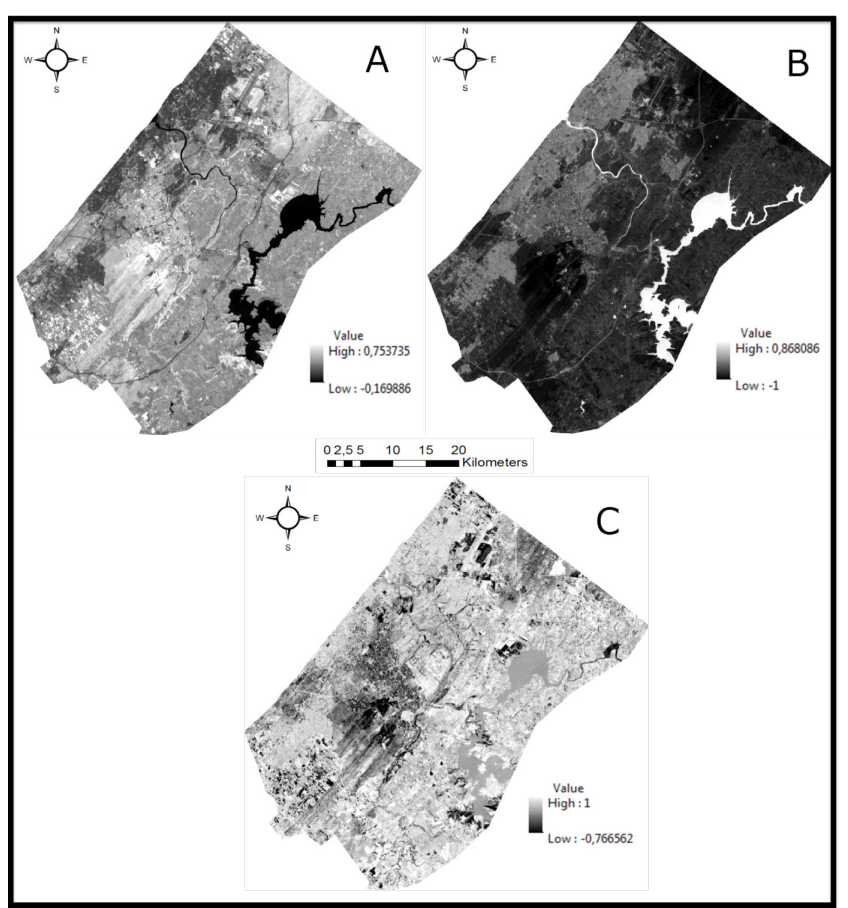

Figure 5. Result of calculation of the three thematic indices, A) SAVI index, B) MNDWI index and C) NDBI index.

that built-up areas are reflected in the SWIR band (band 6), vegetation in NIR band (band 5) and in (Figure 4b) expressed thermal band, whose buit up areas has the highest temperature level.

The input data is now ready to calculate the derived indices. However, we calculated the 3 thematic indices using Equations 2, 3 and 4, results are shown in (Figure 5). The analysis of the indices shows that the contrast of NDBI image showed in (Figure 5a) is not good as those of SAVI and MNDWI images (Figures $5 \mathrm{~b}$ and $5 \mathrm{c}$ ), because many pixels values of vegetation and water areas having positive values close to NDBI values and show gray means tones because they have a noise mixed pixels with buit up characteristics. Reference ${ }^{47}$ used the NBI to extract urban areas in the city of Xian in China and he got a low accuracy of $78.7 \%$. A similar situation was also encountered in this study (see below). We suggest that the characteristics of built-up areas could not be extracted based only on NDBI image. It is for this reason we combine SAVI and MNDWI with NDBI to extract more accurate constructed surfaces. This combination can remove noise of the vegetation and water and thus to improve the extraction accuracy by adding other distinguishing characteristics, such as spatial enhancement of the input data and the thermal bands in the calculation of modified index.

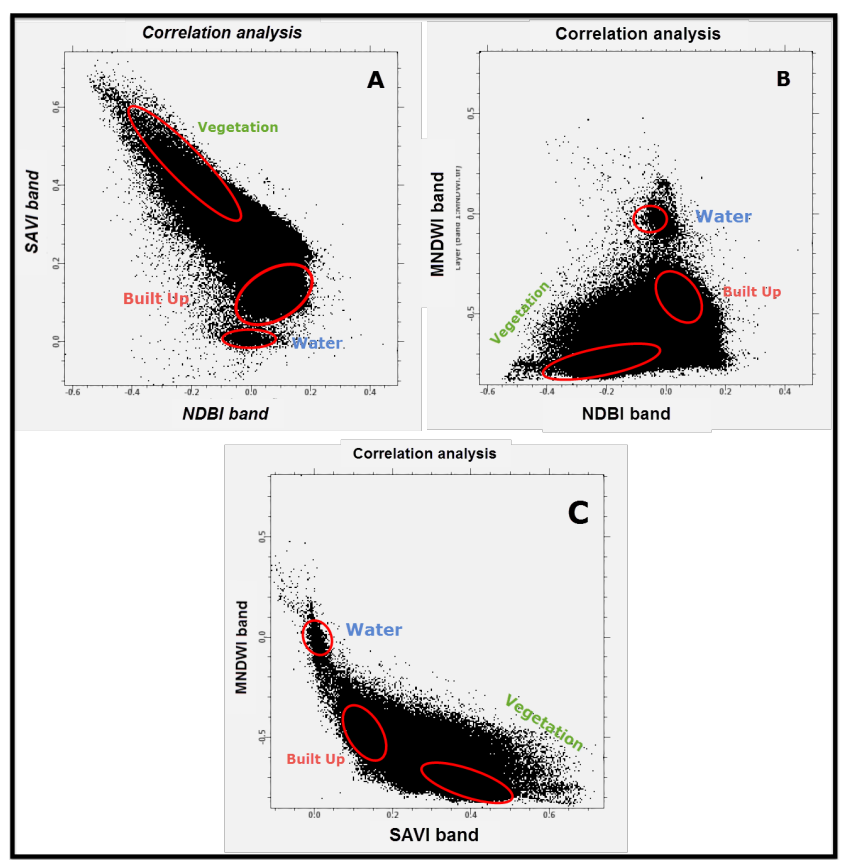

Figure 6. Correlation between derived bands, A) NDBI in $\mathrm{X}$ axis and SAVI band in $\mathrm{Y}$ axis, B) NDBI in $\mathrm{X}$ axis and MNDWI band in $Y$ axis, and $C$ ) SAVI in $X$ axis and MNDWI band in $\mathrm{Y}$ axis.

\subsection{Analysis and Development of New Index}

In order to verify the existing relationships between these three new bands, we built a new image using the three thematic bands as a new input data. The change from the multi spectral original data to the new derived and thematic image can greatly reduce noise between the original multi spectral bands. The correlation analysis showed that the thematic bands are negatively correlated with each other, of which (Figure 6) shows the relationships between the bands 1 . Correlation between NDBI and SAVI, 2. Correlation between NDBI and MNDWI and 3. Correlation between MNDWI and SAVI.

A unique feature that can be drawn from the correlation analysis is that the average value of constructed surfaces is greater than the water and vegetation in the NDBI band. In addition, the average value of constructed surfaces in NDBI band surmounts its values in the SAVI and MNDWI bands. To simplify the relashionships analysis, (Figure 7) shows a graph demonstrated with the calculation of basic statistics in each index.

In the case of Landsat $8 \mathrm{OLI}$, there are two mean infrared bands (bands 6 and 7) and two thermal (bands 


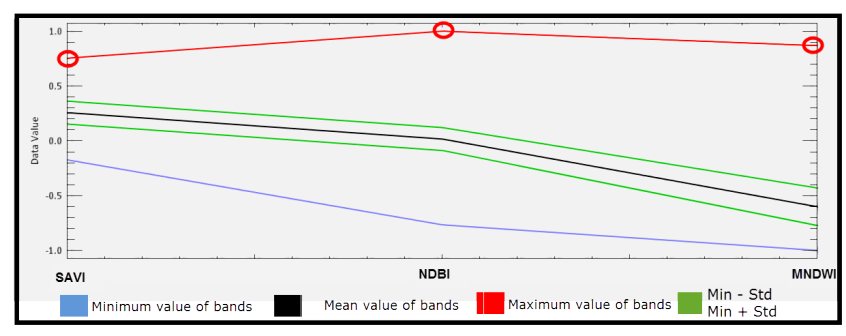

Figure 7. Basic statistics of the three derived indices showing bands reflectivity of every derived index, band 1: SAVI, band 2: NDBI and band 3 MNDWI.
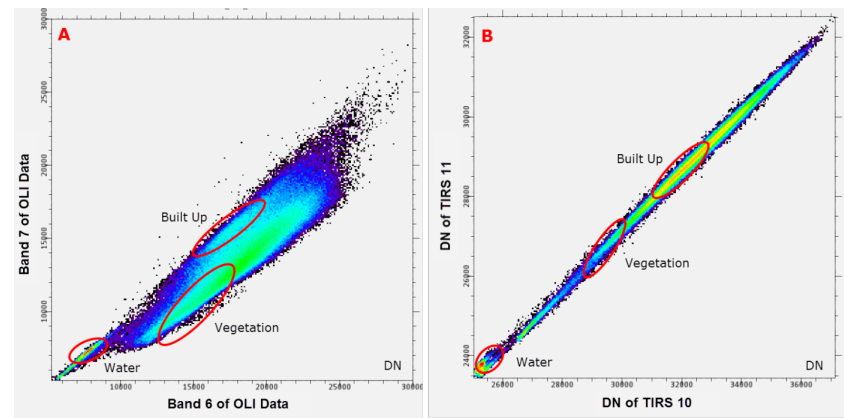

Figure 8. Correlation between SWIR bands 6 in $\mathrm{X}$ axis and band 7 in $\mathrm{Y}$ axis, B) Correlation between thermal bands 10 in $\mathrm{X}$ axis and band 11 in $\mathrm{Y}$ axis.

10 -11). Our analysis has shown that there is a strong positive correlation between the average infrared bands (Figure 8a) and a strong positive correlation between thermal bands 10 and 11 (Figure 8b). This information has allowed us to use principal component analysis - PCA in order to have all most of the information in all groups represented by the variance that can be compressed into a small number of bands with less loss of information. PCA is a technique used to highlight variations and to highlight the strong models in the dataset. It is often used to make the data easy to explore and visualize. The technique allows unnecessary data to be compacted within a few bands so that the dimensionality of the data is diminished.

To calculate SAVI and MNDWI we used Equations 3 and 4 . On the other side, we used a particular expression to calculate NDBI, which allowed us to combine thermal and spectral data into a single index. PCA process was used to generate the new NDBI image, we reduced information using PCA in order to modify the conventional NDBI by using the new combination which is expressed by the Equation 9, improving the NDBI extraction results, by changing the classical index, changes are based on the complemnetary information added by the effect of thermal bands and their important roles in the detection and characterization of constructed surfaces. The purpose of this amendment is to increase the accuracy of extraction of built-up areas and reduce confusion between built up and non-built surfaces in continious data; in other worlds, highlight the membertship of pixels that represent buit up areas in the modified index.

$$
\begin{gathered}
N D B I_{\text {TIRS }}= \\
\frac{P C A(b 6, b 7)+P C A(b 10, b 11)-b \mathbf{5}}{P C A(b 6, b 7)+P C A(b 10, b 11)+b 5}
\end{gathered}
$$

The calculation of the modified index is made by the transformation to "unsigned integer" values of thermal bands in order to comply with the type values used for the multi spectral bands. Since the modified formula of NDBI has positive values and to reduce the noise, we make a subtraction of SAVI and MNDWI indices from NDBI using (Equation 10), it should be noted that the division by tow in the last Equation (10) is used to eliminate negligible values.

$$
\frac{\begin{array}{c}
\text { Enhanced Built Up Index } \\
(S A V I+M N D W I)
\end{array}}{2}=
$$

\subsubsection{Tresholding}

Continuous image composed mainly by positive values whose built up surfaces has a specified range that can easily categorize constructed surfaces. Thresholding techniques can classify the grayscale image into tow classes. In this study, tresholding process is done by using an automatic binarisation method based on histogram frequency. Otsu method ${ }^{48}$ is a data binarization algorithm classification into two classes. This method is based only on the frequency histogram, is the proportion of gray level pixels. The principle is to find the threshold that minimizes the intraclass variance (' $w$ ' = Within) and weighted pixel (Equation 11).

$$
\left.\sigma_{\downarrow} w^{\dagger} 2(t)=q_{\downarrow} 1(t) \sigma_{\downarrow} 1^{\dagger} 2(t)+q_{\downarrow} 2(t) \sigma_{\downarrow} 2^{\dagger} 2 t\right)
$$

With $t \in[0 ; 255]$ the threshold of separation of two classes. The quantities $q 1 \mathrm{t}$ ) and $\mathrm{q} 2 \mathrm{t}$ ) represent the relative proportions of the two classes $q 2 \mathrm{t}$ ) $=1-\mathrm{q} 1 \mathrm{t}$ )).

The algorithm is programmed only from the normalized histogram i.e. frequencies) of the image, not the image itself fast algorithm). To further accelerate the calculations, the relationship between the variances intra 


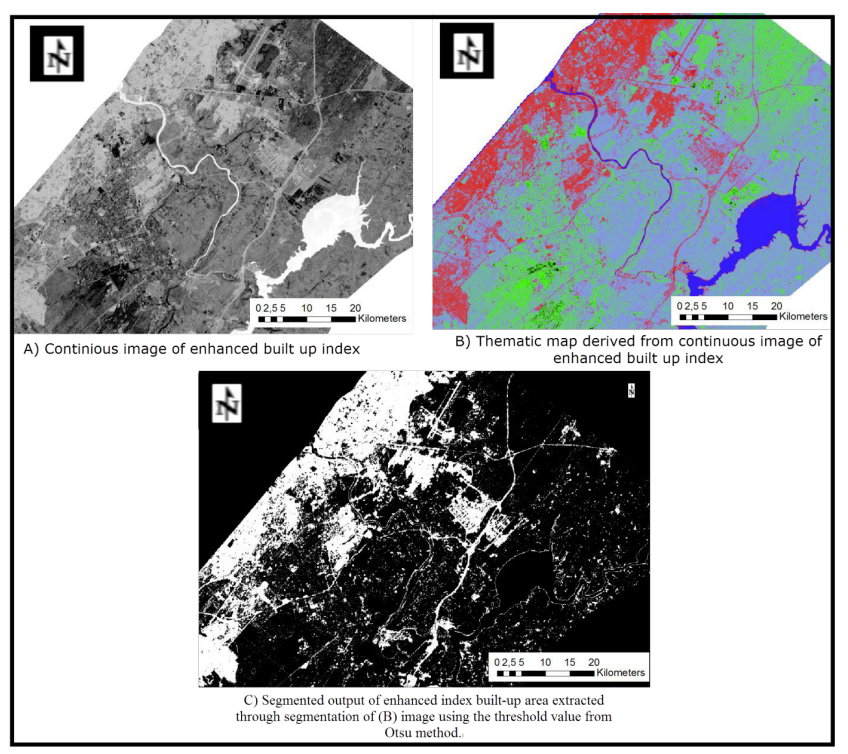

Figure 9. Map showing A) Continuous image of built-up areas, B) Thematic map derived from continuous image, and C) Segmented output of final result built-up area extracted through segmentation of the image using O'tsu method.

and inter-class can be used. The total average image $\mu$ is written, whatever $\mathrm{t}$ in $[0 ; 255]$ (Equation 12),

$$
\mu=q_{1}(t) \mu_{1}(t)+q_{2}(t) \mu_{2}(t)
$$

\section{Results and Discussion}

Figure 9 shows the final result of a binary image (Figure 8C) derived from continuous image (Figure 8A) while, (Figure $8 \mathrm{~B}$ ) shows a colored image of our enhanced index result.

We validate the accuracy of output image by the generation of confusion matrix, a high resolution image was used to validate the overall accuracy of our results, we used 300 random points in the image as a reference points. We compared our results with other indices such as the classic NDBI and Index-based index built up (IBI) proposed by ${ }^{19}$, results are expressed in Table 2.

Our enhanced index showed an improved level and a high accuracy of the membership between buit up areas and other land cover types (Table 2), the improvement is generally due to the spatial enhancement of the pixels in order to increase the membership of built up areas with a distinctive value during the binarization of the resulting image. Indeed, integration of thermal bands makes it possible to optimize the high reflectivity of the medium
Table 2. Confusion matrix

\begin{tabular}{|c|c|c|c|c|}
\hline \multicolumn{5}{|c|}{ Reference Data } \\
\hline $\begin{array}{l}\text { Classified Data } \\
\text { NDBI method }\end{array}$ & $\begin{array}{l}\text { Built- } \\
\text { up } \\
\text { area } \\
\end{array}$ & $\begin{array}{l}\text { Non- } \\
\text { built- } \\
\text { up area }\end{array}$ & $\begin{array}{c}\text { Classified } \\
\text { total }\end{array}$ & $\begin{array}{c}\text { User's } \\
\text { accuracy } \\
\%)\end{array}$ \\
\hline Built-up area & 52 & 24 & 76 & 64,29 \\
\hline Non-Built_up area & 47 & 177 & 224 & 95,65 \\
\hline Reference total & 129 & 171 & 300 & \\
\hline $\begin{array}{c}\text { Producer's } \\
\text { accuracy \%) }\end{array}$ & $44,50 \%$ & $98,02 \%$ & & \\
\hline $\begin{array}{l}\text { Overall accuracy } \\
\text { NDBI method }\end{array}$ & \multicolumn{4}{|c|}{$76,33 \%$} \\
\hline \multicolumn{5}{|l|}{$\begin{array}{c}\text { Classified Data } \\
\text { IBI Index) }\end{array}$} \\
\hline Built-up area & 30 & 10 & 40 & $75 \%$ \\
\hline Non-built-up area & 217 & 43 & 260 & $83 \%$ \\
\hline Reference total & 73 & 227 & 300 & \\
\hline $\begin{array}{c}\text { Producer's accuracy } \\
\%)\end{array}$ & $41 \%$ & $95 \%$ & & \\
\hline $\begin{array}{c}\text { Overall accuracy IBI } \\
\text { index }\end{array}$ & \multicolumn{4}{|c|}{$82,33 \%$} \\
\hline $\begin{array}{c}\text { Classified } \\
\text { Data modified, } \\
\text { thematic NDBI } \\
\text { index } \\
\end{array}$ & & & & \\
\hline Built-up area & 5 & 29 & 34 & $85,29 \%$ \\
\hline Non-built-up area & 32 & 234 & 266 & $87,97 \%$ \\
\hline Reference total & 61 & 239 & 300 & \\
\hline Producer's accuracy & $47,54 \%$ & $97,91 \%$ & & \\
\hline $\begin{array}{l}\text { Overall accuracy } \\
\text { Modified thematic } \\
\text { index }\end{array}$ & \multicolumn{4}{|c|}{$87,67 \%$} \\
\hline
\end{tabular}

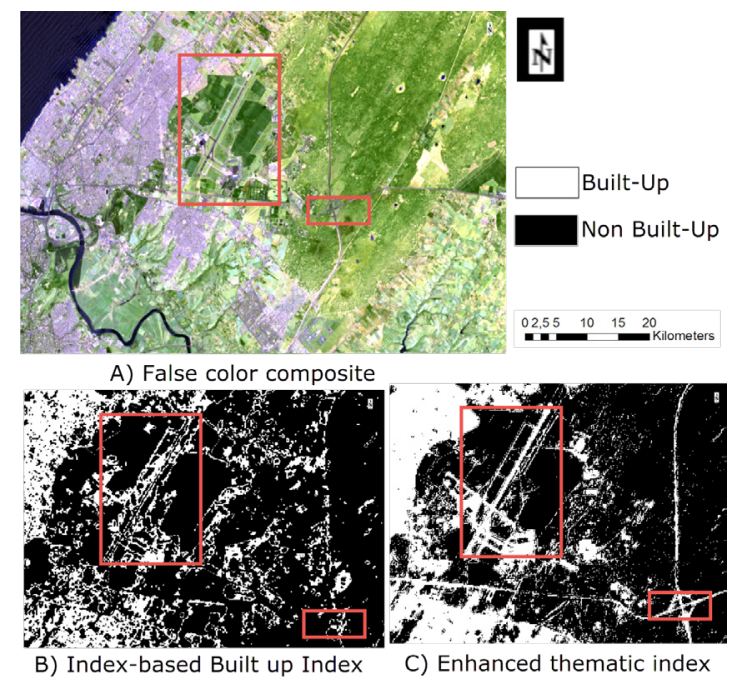

Figure 10. Comparison of the results from the classical IBI approach and enhanced thematic built up index at a sample location Red rectangles in each panel show the places where the IBI approach wrongly extracted built up areas as a constructed surface). 
infrared bands for built up index, by using modified NDBI in Equation 10.

Figure 10 shows a comparison between extraction results obtained from the IBI index and our enhanced index in a binary image, improvements are seen in the road network of the paved areas in which shows the airport of Sale city that is fully extracted using our thematic enhanced index against the IBI index, contrariwise the noise exists in the extraction of IBI value, at the level of the boundary area in red showing the extraction of highways which is extracted accurately using our approach.

\section{Conclusion}

Using multispectral data to extract built up areas can effectively fulfill the need of automatic extraction, spectral indices have the ability to extract objects with high accuracy if other complementary features used. Our research showed that urban heat island effect and spatial enhancement of the data, can easily enhance the accuracy of constructed surfaces. The approach showed a remarkable extraction of built up areas using moderate resolution of Landsat 8 data.

\section{References}

1. Wu C. Normalized spectral mixture analysis for monitoring urban composition using ETM+ imagery. Remote Sensing of Environment. 2004; 93(4):480-92.

2. Smith AJ. Subpixel estimates of impervious surface cover using Landsat TM imagery. [MS Scholarly Paper]. College Park: Department of Geography, University of Maryland; 2000.

3. Zhao $\mathrm{H}$, Xiaoling C. Use of normalized difference bareness index in quickly mapping bare areas from TM/ETM+. Proceedings of 2005 IEEE International Geoscience and Remote Sensing Symposium, IGARSS ‘05; 2005 Jul 25-29. p. $1666-8$.

4. Weng Q. Remote sensing of impervious surfaces in the urban areas: Requirements, methods and trends. Remote Sensing of Environment. 2012; 117:34-49.

5. Ridd MK. Exploring a VIS (Vegetation-Impervious surfaceSoil) model for urban ecosystem analysis through remote sensing: Comparative anatomy for cities. International Journal of Remote Sensing. 1995; 16(12):2165-85.

6. Quackenbush LJ. A review of techniques for extracting linear features from imagery. Photogrammetric Engineering and Remote Sensing. 2004; 70(12):1383-92.
7. Richards J, Landgrebe D, Swain P. A means for utilizing ancillary information in multispectral classification. Remote Sensing of Environment. 1982; 12(6):463-77.

8. Vogelmann J, Sohl T, Campbell P, Shaw D. Regional land cover characterization using Landsat Thematic mapper data and ancillary data sources. Environmental Monitoring and Assessment. 1998; 51(1-2):415-28.

9. Wu C, Murray AT. Estimating impervious surface distribution by spectral mixture analysis. Remote Sensing of Environment. 2003; 84(4):493-505.

10. Turner DP, Cohen WB, Kennedy RE, Fassnacht KS, Briggs JM. Relationships between leaf area index and Landsat TM spectral vegetation indices across three temperate zone sites. Remote Sensing of Environment. 1999; 70(1):5268.

11. Zha Y, Gao J, Ni S. Use of normalized difference built-up index in automatically mapping urban areas from TM imagery. International Journal of Remote Sensing. 2003; 24(3):583-94.

12. Rogers A, Kearney M. Reducing signature variability in unmixing coastal marsh Thematic Mapper scenes using spectral indices. International Journal of Remote Sensing. 2004; 25(12):2317-35.

13. Chen J, Li M, Liu Y, Shen C, Wei W. Extract residential areas automatically by new built-up index. 18th International Conference on Geoinformatics; 2010 Jun 18-20.

14. Zhang Q, Wang J, Peng X, Gong P, Shi P. Urban built-up land change detection with road density and spectral information from multi-temporal Landsat TM data. International Journal of Remote Sensing. 2002; 23(15):3057-78.

15. Guindon B, Zhang Y, Dillabaugh C. Landsat urban mapping based on a combined spectral-spatial methodology. Remote Sensing of Environment. 2004; 92(2):218-32.

16. Al-Sharif AA, Pradhan B, Shafri HZM, Mansor S. Spatiotemporal analysis of urban and population growths in Tripoli using remotely sensed data and GIS. Indian Journal of Science and Technology. 2013; 6(8):5134-42.

17. Atif I, Mahboob MA, Waheed A. Spatio-temporal mapping and multi-sector damage assessment of 2014 flood in Pakistan using Remote Sensing and GIS. Indian Journal of Science and Technology. 2016; 9(1).

18. $\mathrm{Xu} \mathrm{H}$. Extraction of urban built-up land features from Landsat imagery using a thematic oriented index combination technique. Photogrammetric Engineering and Remote Sensing. 2007; 73(12):1381-91.

19. $\mathrm{Xu} \mathrm{H}$. A new index for delineating built-up land features in satellite imagery. International Journal of Remote Sensing. 2008; 29(14):4269-76.

20. McFeeters S. The use of the Normalized Difference Water Index (NDWI) in the delineation of open water features. International Journal of Remote Sensing. 1996; 17(7):1425-32. 
21. Wen CY, Chen JK. Multi-resolution image fusion technique and its application to forensic science. Forensic Science International. 2004; 140(2):217-32.

22. Saraf A. IRS-1C-LISS-III and PAN data fusion: An approach to improve remote sensing based mapping techniques. International Journal of Remote Sensing. 1999; 20(10):1929-34.

23. Lwin KK, Murayama Y. Evaluation of land cover classification based on multispectral versus pansharpened landsat ETM+ imagery. GIScience and Remote Sensing. 2013; 50(4):458-72.

24. Pohl C, Van Genderen JL. Review article multisensor image fusion in remote sensing: Concepts, methods and applications. International Journal of Remote Sensing. 1998; 19(5):823-54.

25. Amolins K, Zhang Y, Dare P. Wavelet based image fusion techniques - An introduction, review and comparison. ISPRS Journal of Photogrammetry and Remote Sensing. 2007; 62(4):249-63.

26. Zhang Y. Problems in the fusion of commercial highresolution satelitte as well as Landsat 7 images and initial solutions. International Archives of Photogrammetry Remote Sensing and Spatial Information Sciences. 2002; 34(4):587-92.

27. Shah VP, Younan NH, King RL. An efficient pan-sharpening method via a combined adaptive PCA approach and contourlets. IEEE Transactions on Geoscience and Remote Sensing. 2008; 46(5):1323-35.

28. Tu TM, Su SC, Shyu HC, Huang PS. A new look at IHSlike image fusion methods. Information Fusion. 2001; 2(3):177-86.

29. Jung HS, Park SW. Multi-sensor fusion of Landsat 8 Thermal Infrared (TIR) and Panchromatic (PAN) images. Sensors. 2014; 14(12):24425-40.

30. Jawak SD, Luis AJ. A comprehensive evaluation of PANsharpening algorithms coupled with resampling methods for image synthesis of very high resolution remotely sensed satellite data. Advances in Remote Sensing. 2013; 2(4):332-44.

31. Oke TR. The energetic basis of the urban heat island. Quarterly Journal of the Royal Meteorological Society. 1982; 108(455):1-24.

32. Zhangyan J, Yunhao C, Jing L. On urban heat island of Beijing based on Landsat TM data. Geo-Spatial Information Science. 2006; 9(4):293-7.

33. Tran H, Uchihama D, Ochi S, Yasuoka Y. Assessment with satellite data of the urban heat island effects in Asian mega cities. International Journal of Applied Earth Observation and Geoinformation. 2006; 8(1):34-48.

34. Azmi R, Saadane A, Kacimi I. Estimation of spatial distribution and temporal variability of land surface temperature over Casablanca and the surroundings of the city using different Landat satellite sensor type (TM, ETM+ and OLI). International Journal of Innovation and Applied Studies. 2015; 11(1):49-57.

35. Zhang Y, Odeh IOA, Han C. Bi-temporal characterization of land surface temperature in relation to impervious surface area, NDVI and NDBI, using a sub-pixel image analysis. International Journal of Applied Earth Observation and Geoinformation. 2009; 11(4):256-64.

36. Falahatkar S, Hosseini SM, Soffianian AR. The relationship between land cover changes and spatial-temporal dynamics of land surface temperature. Indian Journal of Science and Technology. 2011; 4(2):76-81.

37. Kumar D, Shekhar S. Statistical analysis of land surface temperature-vegetation indexes relationship through thermal remote sensing. Ecotoxicology and environmental safety. 2015; 121:39-44.

38. Plan HCa. RGPH 2014. Haut commissariat au plan 2014. Available from: http://www.hcp.ma/downloads/RGPH2014_t17441.html

39. Chavez PS. Image-based atmospheric corrections-revisited and improved. Photogrammetric Engineering and Remote Sensing. 1996; 62(9):1025-35.

40. Sobrino JA, Jimenez-Munoz JC, Paolini L. Land surface temperature retrieval from LandsaT TM 5. Remote Sensing of environment. 2004; 90(4):434-40.

41. Johnson B. Effects of pansharpening on vegetation indices. ISPRS International Journal of Geo-Information. 2014; 3(2):507-22.

42. Matsuoka M. Comparison of the spectral properties of pansharpened images generated AVNIR-2 and PRISM Onboard ALOS. ISPRS Annals of the Photogrammetry, Remote Sensing and Spatial Information Sciences. 2012; 7:291-6.

43. Vrabel J. Multispectral imagery band sharpening study. Photogrammetric Engineering and Remote Sensing. 1996; 62(9):1075-84.

44. Laben CA, Brower BV. Process for enhancing the spatial resolution of multispectral imagery using pansharpening. Google Patents; 2000.

45. Siddiqui $Y$, The modified IHS method for fusing satellite imagery. ASPRS 2003 Annual Conference Proceedings; Anchorage, Alaska. 2003..

46. Sun W, Chen B, Messinger DW. Nearest-neighbor diffusion-based pansharpening algorithm for spectral images. Optical Engineering. 2014; 53(1):013107.

47. Hong'an W, Jianjun J, Jie Z. Dynamics of urban expansion in Xi'an City using Landsat TM/ETM+ data. Acta Geographica Sinica. 2005; 60(1):143-50.

48. Otsu N. A threshold selection method from gray-level histograms. Automatica. 1975; 11(285-296):23-7. 